\title{
A One-dimensional Model for Prediction of Breakout Noise from a Finite Rectangular Duct with different Acoustic Boundary Conditions
}

\author{
B. Venkatesham*, Ardhendu G. Pathak* and M. L. Munjal ${ }^{\dagger}$ \\ Facility for Research in Technical Acoustics, Department of Mechanical Engineering, \\ Indian Institute of Science, Bangalore 560012, India
}

(Received 30 March 2007; accepted 29 June 2007)

\begin{abstract}
This paper describes a simple one-dimensional predictive model for breakout noise from a thin rectangular duct with different end conditions like anechoic termination, rigid-end, and open-end termination. This model incorporates acoustic reflection effects in the duct internal sound field by using standing wave pattern with the transfer matrix approach. The coupling phenomenon is modelled making use of a common axial wave number for acoustic waves inside and flexural waves in the walls of the duct. The noise radiated from the rectangular duct is modelled as a finite-length line source. This standing wave analytical model has been compared with numerical model, the corresponding progressive wave model, and experimental results available in the literature. The effect of coupling in acoustic modes and flexural modes has been discussed.
\end{abstract}

${ }^{\dagger}$ Member of the International Institute of Acoustics and Vibration (IIAV)
${ }^{*}$ GE, Global Research, Bangalore, India

\section{INTRODUCTION}

Heating, ventilation, and air-conditioning (HVAC) systems consist of large air-handling units with thin flexible ducts of different geometrical shapes and sizes connected in parallel and series. The noise generated by air-handling units propagates through the ducts in axial and transverse directions. Noise radiation in the transverse direction, due to secondary radiation of the duct walls excited by the internal sound field, is called breakout noise. ${ }^{1,2}$ Cummings ${ }^{3}$ provided an excellent research review on breakout and break-in noise in HVAC ducts and pointed out the gaps existing in current research and future directions for the prediction of breakout noise.

Cummings ${ }^{2}$ modelled the internal sound field of a forward progressive wave by considering an infinite duct. He modelled the coupling phenomena between the duct acoustic field and flexural waves along flexible duct wall by using a common axial wave number. The external acoustic radiation from duct walls has been estimated in the literature: namely, a finite length line source model, ${ }^{1,2}$ equivalent cylindrical source of finite length, ${ }^{5}$ and a finite element method. ${ }^{6}$ Generally, air-handling units generate most of the sound at low frequencies where the coupling phenomenon is strong. So, any worthwhile analytical solution should be very accurate in the low frequency region. Cummings presented a simple analytical formulation to predict the transverse transmission loss $\left(\mathrm{TL}_{t p}\right)$ based on progressive waves and mentioned the research requirements for acoustic reflection and structural reflection effects in the prediction of breakout noise..$^{1-3}$

In the present paper, acoustic reflections have been incorporated by using the transfer matrix method. ${ }^{4}$ Effects of the finite length of the duct as well as the boundary conditions on the pressure distribution are considered by using the standing wave pattern. Three types of boundary conditions, namely anechoic terminations, rigid-end and open-end terminations are studied in this paper. This methodology can however be extended to any type of boundary conditions like an abrupt change in the cross section of the duct, etc. An anechoic boundary condition with a standing wave pattern is equivalent to the progressive wave approach used by Cummings in the prediction of breakout noise. Computation of the axial wave number requires an iterative approach with the possibility of ill-conditioning in the result. This problem is overcome by rearranging the equation in matrix form.

A numerical model has been developed using a commercial package SYSNOISE to validate the proposed analytical model. Coupled, as well as decoupled, numerical analysis has been carried out to study the effect of coupling on the applicable frequency range and computational burden.

\section{ACOUSTIC WAVE EQUATION}

Figure 1 shows the schematic diagram of a rectangular duct with its dimensions. One can assume strong coupling between the duct cavity and the flexible plate, and relatively weak coupling between the flexible plate and the outside radiated acoustic field. Consider a co-ordinate system as shown in Fig. 2 around the duct perimeter and in the axial direction. The governing equations in this co-ordinate system are as follows: ${ }^{1,9}$

Mass Continuity Equation:

$$
\rho_{i} \frac{\partial u_{x}}{\partial x}+\frac{p L \bar{\beta}}{c_{i} S}+\frac{\partial \rho}{\partial t}=0
$$

Momentum Equation:

$$
\rho_{i} \frac{\partial u_{x}}{\partial t}+\frac{\partial p}{\partial x}=0
$$

where $\rho_{i}$ and $c_{i}$ are mean values of density and sound speed of the medium inside the duct, $p$ and $\rho$ are acoustic perturba- 